\title{
summary
}

\section{Implants provide better health-related quality of life than conventional treatment for edentulous patients}

Measuring the effect of intra-oral implant rehabilitation on health related quality of life in a randomised controlled trial. Awad MA, Locker D, Korner-Bitensky N, Feine JS. J Dent Res 2000; 79:1659-1663

Question: Does use of dental implants in edentulous patients result in better quality of life compared with conventional treatment?

Objective To compare the effect on health-related quality of life of two treatments for edentulism, conventional mandibular dentures and implant-supported prostheses.

Design A randomised controlled trial in a hospital setting.

Intervention A total of 102 people were assigned randomly into either implant or conventional treatment groups. The implant group received two implants in the lower canine region connected by a short bar. Either a conventional denture or over denture was provided in the lower jaw with conventional upper denture. Patients were analysed using the intent-to-treat principle, with patients who withdrew being allocated their pretreatment OHIP scores

Outcome measures An oral health impact profile (OHIP) was conducted at the initial appointment and 2 months after the fitting of the prosthesis.

Results See Table 1.
Conclusions Using a validated standard assessment tool, this trial suggests that patients who had implant treatment experienced more improvements in perceived oral health than patients having conventional treatment.

Acknowledgements Nobel Biocare Canada, Laboratoire Dentachrome, Dentsply Canada, the Canadian MRC, Health and Welfare Canada (NHRDP) and Fonds de Recherché en Santé du Québec.

Evidence-Based Dentistry (2002) 3, 73-74. doi:10.1038/ sj.ebd.6400115

Address for reprints: JS Feine, McGill University, 3640 University Street, Montreal, Quebec H3A 2B2, Canada. E-mail: jocelyne.feine@mcgill.ca

Table 1 OHIP scores for treatment after implants or conventional treatment*

\begin{tabular}{|c|c|c|c|c|}
\hline \multirow[b]{2}{*}{ Domains } & \multicolumn{2}{|c|}{ Mean score implant group $(n=54)$} & \multicolumn{2}{|c|}{ Mean score conventional group $(n=48)$} \\
\hline & Pre-treatment & Post-treatment & Pre-treatment & Post-treatment \\
\hline Total OHIP score & 100.6 & $66.1 \dagger$ & 98.7 & 89.3 \\
\hline Functional limitation & 20.65 & $14.4 \dagger$ & 24.2 & 18.5 \\
\hline Physical pain & 20.50 & $12.5 \dagger$ & 19.4 & 18.7 \\
\hline Psychological discomfort & 11.96 & $7.09+$ & 11.88 & 10.3 \\
\hline Physical ability & 18.9 & $12.1 \dagger$ & 18.1 & 15.8 \\
\hline Psychological disability & 10.7 & $7.9 \dagger$ & 10.1 & 10.6 \\
\hline Social disability & 6.4 & $5.5 \dagger$ & 6.7 & 7.0 \\
\hline Handicap & 8.2 & $6.7 \dagger$ & 8.4 & 8.3 \\
\hline
\end{tabular}

*Standard deviation available in original article.

$\dagger$ Significant difference between pre- and post-treatment OHIP scores.

OHIP, Oral health impact profile.

\section{Commentary}

There is growing consensus on the need to include patient-centred outcome measures when evaluating treatment, to complement clinical outcome measures. This trial compared the effects on oral health-related quality of life of two treatments for edentu- lism, that is, conventional mandibular dentures and implant-supported prosthesis. The findings indicate that in the short term (after a 2-month follow-up) implant treatment is significantly associated with a greater reduction in oral health impact (negative oral health-related quality of life) compared with conventional therapy.

The measure of oral health-related quality of life was the OHIP which seeks to measure the burden of oral disease or ill health on life quality. Using different measures of oral health-related quality of life (which incorporate both positive 
and negative dimensions) it may be possible to assess oral health gain not just in terms of the reduction of the burden of oral ill health brought by implant therapy but also enhancements to life quality.

Thirty-one per cent of the variance was explained in the regression model indicating that pretreatment OHIP score and type of treatment (implants) were the largest predictors of postoperative OHIP scores. Is there a possibility that other important predicators of oral health-related quality of life have not been included, such as ethnic and cultural factors, and influences of personality traits?

The follow-up period was short (2 months) and, as the authors point out, a long-term follow-up would be of benefit. Could this short-term follow-up to determine outcomes be inappropriate? The implant group who undergo surgery are likely to experience a reduced quality of life in the immediate postoperative period (as a result of the surgery) and this could influence their perception of how their oral health impacts on their life quality when assessed a short time after — in a similar way that people may feel euphoric about their health after recovering from a recent illness. We await the long-term follow-up.

Colman McGrath Periodontology and Public Health, Faculty of Dentistry, University of Hong Kong, Hong Kong SAR, China 